OPEN ACCESS

Edited by:

Diana Boraschi,

National Research Council, Italy

Reviewed by:

Janos Szebeni,

University of Miskolc, Hungary

Yang $\mathrm{Li}$,

University of Colorado Denver, USA

${ }^{*}$ Correspondence:

Dmitri Simberg

dmitri.simberg@ucdenver.edu

tPresent address: Swetha Inturi,

Noven Pharmaceuticals, Miami, FL, USA

Specialty section: This article was submitted to Inflammation,

a section of the journal

Frontiers in Immunology

Received: 22 November 2016 Accepted: 30 January 2017

Published: 15 February 2017

Citation:

Wang G, Griffin Jl, Inturi S, Brenneman B, Banda NK, Holers VM, Moghimi SM and Simberg D (2017) In Vitro and In Vivo Differences in

Murine Third Complement Component (C3) Opsonization and

Macrophage/Leukocyte Responses to Antibody-Functionalized Iron Oxide Nanoworms.

Front. Immunol. 8:151. doi: 10.3389/fimmu.2017.00151

\title{
In Vitro and In Vivo Differences in Murine Third Complement Component (C3) Opsonization and Macrophage/Leukocyte Responses to Antibody-Functionalized Iron Oxide Nanoworms
}

Guankui Wang', James I. Griffin ${ }^{1}$, Swetha Inturi't, Barbara Brenneman ${ }^{1}$, Nirmal K. Banda ${ }^{2}$, V. Michael Holers ${ }^{2}$, Seyed Moein Moghimi ${ }^{3}$ and Dmitri Simberg ${ }^{1 *}$

\footnotetext{
The Skaggs School of Pharmacy and Pharmaceutical Sciences, University of Colorado Denver, Anschutz Medical Campus, Aurora, CO, USA, ${ }^{2}$ Division of Rheumatology, School of Medicine, University of Colorado Denver, Anschutz Medical Campus, Aurora, CO, USA, ${ }^{3}$ School of Medicine, Pharmacy and Health, Durham University, Queen's Campus, Stockton-on-Tees, UK
}

Balancing surface functionalization and low immune recognition of nanomedicines is a major challenge. Opsonization with the third component of the complement protein (C3) plays a major role in immune cell recognition of nanomedicines. We used dextran-coated superparamagnetic iron oxide nanoworms (SPIO NWs) to study the effect of surface functionalization on C3 opsonization in mouse serum and subsequent macrophage/ leukocyte recognition in vitro as well as on intravenous injection into mice. Previously, we found that in mouse serum, SPIO NWs became opsonized with C3 via complement lectin pathway. Crosslinking the dextran shell with epichlorohydrin significantly decreased C3 opsonization and uptake by mouse peritoneal macrophages. Crosslinked nanoworms (NWs) further functionalized with polyethylene glycol (PEG) or with PEG-antibody (Ab) ( 160 lgG molecules/particle) did not show an increase in C3 opsonization and peritoneal macrophage uptake in vitro. Following tail vein injection into mice, plain crosslinked NWs and PEGylated crosslinked NWs showed very low C3 opsonization and mouse leukocyte uptake. However, Ab-decorated crosslinked NWs showed significant C3 opsonization and high level of complement-dependent uptake by leukocytes in mice. Decreasing the number of conjugated Abs to $46 \mathrm{lgG}$ molecules/particle significantly reduced C3 opsonization and leukocyte uptake. Using fresh mouse lepirudin plasma rather than serum showed better correlation with C3 opsonization in vivo. The reason for this difference could be related to the known instability of complement classical pathway in mouse sera. Our data illustrate that fine-tuning in nanoparticle surface functionalization with Abs is required to avoid excessive complement activation and complementmediated immune uptake in mice, and raise issues with in vitro immunological assays of nanomedicines intended to mimic in vivo conditions.

Keywords: iron oxide, leukocyte, complement, antibody, PEG 


\section{INTRODUCTION}

The success of systemically and locally administered nanomaterials largely depends on the ability of nanosized carriers to efficiently evade the immune system (1). Several pathways of the innate immune system mediate clearance of nanoparticles by phagocytic cells. Complement system is an effector arm of the innate immune system composed of more than 30 blood proteins that accounts for about $5 \%$ of globulins in serum and is responsible for recognizing, eliminating, and destroying pathogens (2). The activation of complement system on foreign surface via lectin, classical, or alternative pathways [LP, classical pathway (CP), or AP, respectively] converges to cleave native $\mathrm{C} 3$ and generate a highly reactive thioester on $\mathrm{C} 3 \mathrm{~b}$, which covalently attaches to reactive functional groups (e.g., hydroxyl and amines) on target surface (3-5). Opsonization by C3b and its cleavage products $(\mathrm{iC} 3 \mathrm{~b}, \mathrm{C} 3 \mathrm{~d}$ ) triggers particle recognition by leukocytes through complement receptors $(6,7)$, whereas soluble cleavage byproducts $\mathrm{C} 3 \mathrm{a}$ and $\mathrm{C} 5 \mathrm{a}$ are among the most potent anaphylatoxins and proinflammatory molecules with low nanomolar affinity (8). Numerous nanomaterials activate the complement system and become opsonized with $\mathrm{C} 3$ in vitro and in vivo (9-13).

Superparamagnetic iron oxide (SPIO) nanoparticles have been used as magnetic resonance imaging (MRI) contrast agents and also as carriers for drug delivery (14). SPIO nanoparticles consist of $5-8 \mathrm{~nm}$ magnetite-maghemite $\left(\mathrm{Fe}_{3} \mathrm{O}_{4}\right.$ and $\left.\gamma-\mathrm{Fe}_{2} \mathrm{O}_{3}\right)$ crystalline cores coated with a polymer (15). Recently, we reported the synthesis of $20 \mathrm{kDa}$ dextran-coated superparamagnetic iron oxide nanoworms (SPIO NWs) with high transverse relaxivity $\mathrm{r} 2$, which makes them promising MRI contrast agents (16). Unfortunately, SPIO NWs potently activate complement in both mice and humans (17). Previously, we demonstrated that mouse complement activation is via LP (17), whereas human complement activation is predominantly via the AP (18). As shown in Figure 1, initiation of the LP starts with the binding of mannose-binding lectin (MBL)-A/C, ficolins, or collectin-11 to the carbohydrates on the pathogen surfaces. The binding leads to activation of MBL-associated serine proteases (MASPs), leading to formation of the complement convertase $\mathrm{C} 4 \mathrm{bC} 2 \mathrm{a}$, cleavage of $\mathrm{C} 3$, deposition of initial $\mathrm{C} 3 \mathrm{~b}$, and possible amplification via the alternative pathway convertase C3bBb. MASP-2 plays a direct role in formation of the complement convertase C4bC2a (19) whereas MASP-1 and MASP-2 indirectly activate MASP-2 (20) and factor $\mathrm{D}(21,22)$, respectively.

Interestingly, modifying the surface dextran coating with epichlorohydrin (ECH) [resulting in poly-(2-hydroxypropyl ether) hydrogel] blocked mouse complement C3 opsonization and leukocyte uptake (16). At the same time, it is not clear how further surface functionalization of ECH-crosslinked NWs (hereafter CL-NWs) affects complement activation and immune uptake. In view of the remarkable redundancy of pathways responsible for immune recognition (7) maintaining the delicate balance between surface functionalization and stealth properties could be a challenging task. In particular, the effect of addition of targeting ligands on "stealth" nanoparticles on complement activation and immune uptake has not been investigated in depth. In order to understand the impact of surface functionalization of CL-NWs with targeting antibodies (Abs) on complement activation and immune recognition, we modified CL-NWs with a polyethylene glycol (PEG) linker followed by a model anticancer $\mathrm{Ab}$ (trastuzumab). Here, we demonstrate that surface modification of CL-NWs that have low complement activation with trastuzumab can increase complement activation dependent on Ab surface

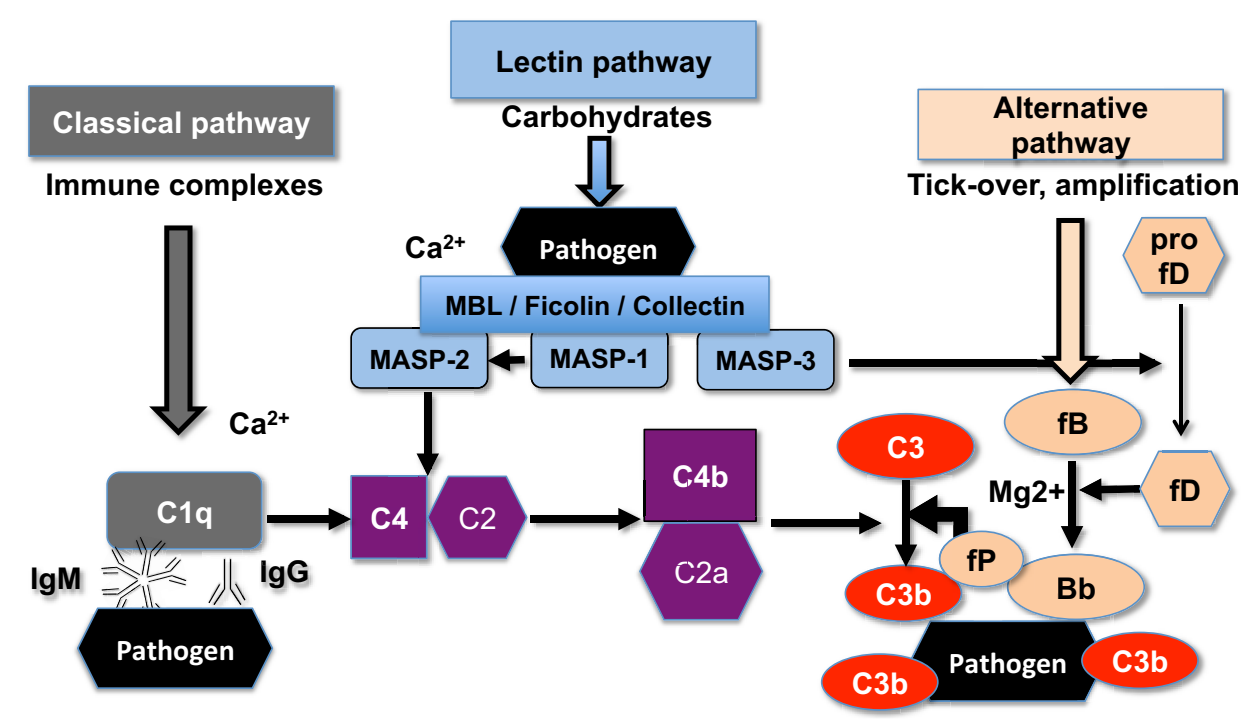

FIGURE 1 | The scheme of the upstream mouse complement pathways. C5a convertase and the terminal membrane attack complex pathway are not shown. There are three initiating complement pathways: classical, lectin, and alternative. All the pathways generate activated central complement protein component C3, liberating C3b, and its fixation on the pathogen (or nanoparticle) surface. 
density, and further raise an issue of in vitro versus in vivo correlation assays of immune recognition of nanoparticles.

\section{RESULTS AND DISCUSSION}

\section{Effect of Nanoworm (NW) Modification and Functionalization on C3 Opsonization and Immune Uptake in Mouse Sera In Vitro}

Dextran-coated SPIO NWs were prepared from $20 \mathrm{kDa}$ dextran, $\mathrm{FeCl}_{2}$, and $\mathrm{FeCl}_{3}$ by a modified Molday and MacKenzie precipitation protocol (23). According to transmitted electron microscopy (Figure 2A), nanoparticle cores contained wormlike aggregates of iron oxide crystals. On the outside, the cores are covered with a shell of dextran chains (Figure 2B). Dextran shell was crosslinked with $\mathrm{ECH}$ in the presence of $\mathrm{NaOH}$ to yield hydrogel-coated CL-NWs (Figure 2B). The residual epoxides on CL-NWs were treated with ammonia to generate primary amines that were further functionalized with heterobifunctional maleimide (MAL)-PEG ${ }_{3400}$-succinimidyl valerate (SVA) to yield CL-NWs-PEG-MAL (hereafter CL-NWs-PEG; Figure 2B). PEG-MAL-functionalized particles were modified with thiolated Ab trastuzumab (Herceptin ${ }^{\circledR}$ ) to yield CL-NWs-PEG-Ab (Figure 2B). According to size measurements (Figure 2C, top), the modifications did not affect the hydrodynamic diameter of NWs. Zeta potential values were slightly negative for SPIO NWs and CL-NWs, but expectably became positive for CL-NWs treated with ammonia and the corresponding functionalized particles (Figure 2C, bottom). Quantitative measurements (see Materials and Methods) showed that on average there were $\sim 160$ IgG molecules per each CL-NWs-PEG-Ab nanoparticle.

In order to confirm the role of the LP as the inciting pathway of complement activation in mouse serum, SPIO NWs were incubated in validated sera obtained from mice deficient for various complement pathways (Figure 1), washed, and analyzed for mouse C3 in Western blotting (the same amount of particles were used in the assay and loaded on the gel). According to Figure 3, SPIO NWs showed strong C3 (C3b/iC3b) opsonization in wild type (WT) mouse serum, whereas the opsonization was blocked in sera deficient for MBL-A/C (LP) and MBL-A/C/factor $\mathrm{D}$ (LP and AP). In addition, C3 opsonization was blocked in sera deficient for factor D and factor B (AP). These data confirm that complement is triggered by MBL/MASP-2-dependent LP activation, whereas the AP provides the amplification loop.

Next, we compared the efficiency of C3 opsonization of CL-NWs, CL-NWs-PEG, and CL-NWs-PEG-Ab with SPIO NWs. Particles were incubated in normal mouse serum or in sera deficient for the LP factors MBL-A/C and MASP-2, washed, loaded in the same amount on a gel, and analyzed by Western blotting. All CL-NWs formulations showed $80-85 \%$ decrease in C3 opsonization in normal serum compared with SPIO NWs (Figures 4A,B). Moreover, CL-NWs formulations incubated in MBL-A/C-deficient and MASP-2-deficient sera (Figures 4A,B) showed further reduction of $\mathrm{C} 3$ opsonization. These data demonstrate that crosslinking predominantly blocks complement activation and functionalization of CL-NWs with PEG or PEG-Ab does not enhance complement activation in mouse serum.

C3 is the critical opsonin mediating the uptake of foreign pathogens by macrophages and leukocytes (2). We tested whether functionalization of CL-NWs affected the uptake by non-activated mouse peritoneal macrophages in mouse serum. As shown in Figure S1 in Supplementary Material, over 70\% of freshly isolated cells showed expression of CD11b (receptor for $\mathrm{iC} 3 \mathrm{~b}$ ) and F4/80 (a macrophage marker). SPIO NWs, CL-NWs, CL-NWs-PEG, and CL-NWs-PEG-Ab were preincubated in normal (WT) mouse serum for $15 \mathrm{~min}$, and then added to the

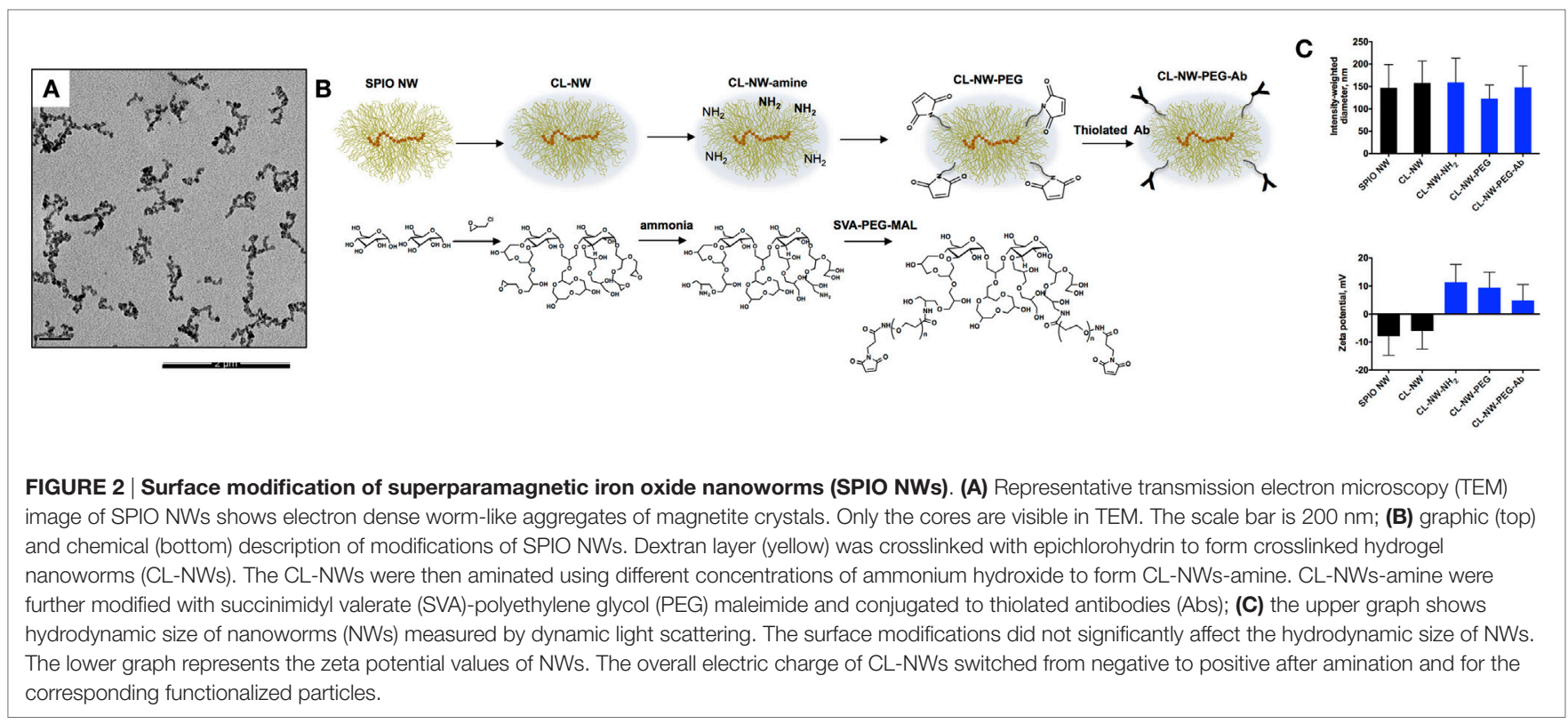




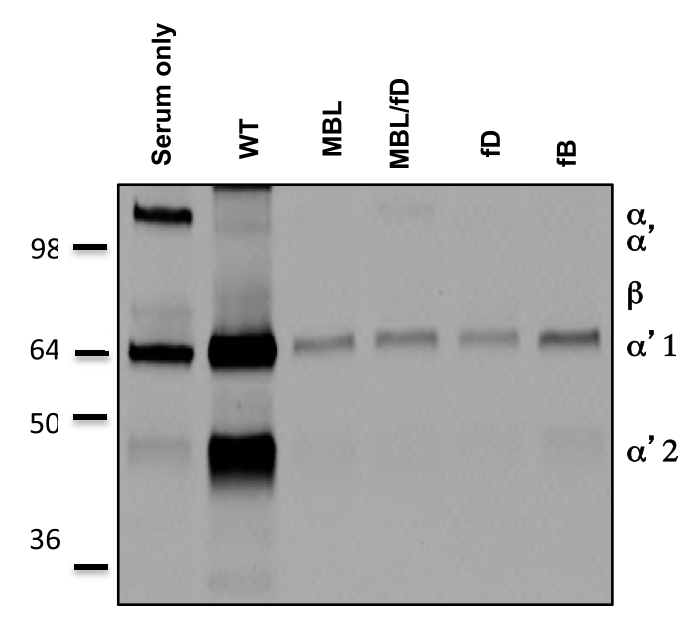

FIGURE 3 | Mechanisms of complement C3 opsonization by non-modified superparamagnetic iron oxide nanoworms (SPIO NWs) Detection of C3 (mostly in iC3b form due to appearance of $\alpha 2^{\prime}$ fragment at $\sim 40 \mathrm{kDa}$ ) bound to SPIO NWs in mouse sera. Nanoworms became opsonized in control (wild type) sera but not in sera deficient for mannosebinding lectin (MBL), MBL/factor $\mathrm{D}$, factor $\mathrm{D}$, and factor $\mathrm{B}$, suggesting that the LP is the initiating route and the AP turnover is responsible for amplification. Note that beta-chain of mouse C3 is not highly detectable with this antibody.

cells at $0.1 \mathrm{mg} / \mathrm{mL}$ Fe for $6 \mathrm{~h}$. According to Prussian blue staining and quantification (Figures 5A,B, see Materials and Methods for details), SPIO NWs showed highly intense cytoplasmic accumulation of iron. CL-NWs showed $80 \%$ less uptake than SPIO NWs. The functionalized CL-NWs had the same level of residual uptake as non-functionalized CL-NWs, suggesting that PEG and Ab modifications do not trigger the uptake in mouse serum. Incubation of SPIO NWs and all CL-NWs formulations in C3-deficient mouse serum resulted in a complete blockade of the residual uptake of CL-NWs formulations (Figures 5A,B), suggesting that the uptake of SPIO NWs and the residual uptake of CL-NW formulations are mediated through C3 opsonization.

\section{Effect of Cl-NWs Functionalization on $\mathrm{C} 3$ Opsonization and Immune Uptake In Vivo}

Previously, we demonstrated that complement C3 mediates the uptake of SPIO NWs by blood leukocytes in mice (10). SPIO NWs, CL-NWs-PEG, and CL-NWs-PEG-Ab were injected intravenously into mice at $5 \mathrm{mg} \mathrm{Fe}$ per $\mathrm{kg}$ body weight, recovered from blood 5 min post-injection using a magnetic column [in this process, both free particles and magnetically labeled leukocytes were enriched (10)], and analyzed for C3 opsonization and leukocyte uptake as shown in Figure 6A. We used a previously established $(10,16-18)$ dot-blot procedure to compare the levels of C3 opsonization on NWs. According to Figure S2 in Supplementary Material, C3 dot-blot assay correlates with $\mathrm{C} 3$ Western blot for determining the levels of $\mathrm{C} 3$ on particles. According to Figures 6B,C, SPIO NWs showed high level of C3 opsonization in vivo and leukocyte uptake. Similar to in vitro serum results, CL-NWs-PEG showed less than $10 \%$ of
C3 compared to SPIO NWs, and low level of leukocyte uptake. Non-modified CL-NWs also showed low level of in vivo C3 opsonization and leukocyte uptake (Figure S3 in Supplementary Material), confirming our previous findings (10). However, in contrast to in vitro measurements, CL-NWs-PEG-Ab decorated with $\sim 160 \mathrm{IgG} /$ particle showed $66 \%$ of C3 opsonization of SPIO NWs and high leukocyte uptake in vivo (Figures 6B,C). Injection of CL-NWs-PEG-Ab into C3 knockout (KO) mice that completely lacks $\mathrm{C} 3$ abolished the leukocyte uptake (Figure 6C). These data suggest that conjugation of $\operatorname{IgG}$ on the particles triggered complement activation and complement-dependent immune uptake that was not detected using in vitro assays in mouse serum.

Surface immobilized Abs and immune complexes, however, are known to trigger complement via the CP (24). It is likely that in vivo Ab-functionalized NWs trigger the CP. At least two surface-bound IgG molecules must be bridged by a C1q molecule before activation of the $\mathrm{CP}$ can proceed. In order to test whether the number of IgG per particle can control complement activation in vivo, we synthesized CL-NWs-PEG-Ab bearing different $\mathrm{Ab}$ densities and tested their $\mathrm{C} 3$ opsonization and leukocyte uptake in vivo. According to Figures 7A,B, CL-NWs-PEG-Ab with $1 \mathrm{IgG} /$ particle, $8 \mathrm{IgG} /$ particle, and $46 \mathrm{IgG} /$ particle showed significantly lower levels of in vivo C3 opsonization (17, 3, and $22 \%$ of SPIO NWs, respectively) and leukocyte uptake than CL-NWs-PEG-Ab with $160 \mathrm{IgG} /$ particle. However, the observation of higher C3 opsonization with CL-NW-PEG-Ab bearing a single $\mathrm{Ab}$ molecule compared with CL-NW-PEG-Ab with $8 \mathrm{IgG} /$ particle is intriguing. The reason for this is unclear, but this suggests the involvement of other in vivo factors regulating complement activation and fixation and therefore requires further investigation. Nevertheless, these experiments suggest that decreasing surface density of $\mathrm{Ab}$ molecules can suppress complement activation and immune cell recognition in vivo. CL-NWsPEG-Ab with 8 trastuzumab/particle showed specific uptake by HER2/neu + human breast cancer cell line SKBR-3 (Figure S4 in Supplementary Material), suggesting that the immobilized $\mathrm{Ab}$ is functional on the nanoparticles and therefore may bind to its designated target in vivo.

Next, we sought an explanation to the observed lack of complement activation by CL-NWs-PEG-Ab (160 IgG/particle) in serum (Figure 4) versus in vivo. Previous evidence suggested that the CP of the complement system is unstable in mouse sera (25-27) and, furthermore, starts losing its activity at room temperature (RT) and even during $-70^{\circ} \mathrm{C}$ storage (26). Accordingly, blood clotting procedures and serum isolation steps could trigger loss of CP activity and explain poor C3 opsonization in mouse serum through this pathway. To address this, we repeated the experiments with fresh mouse plasma using recombinant hirudin (lepirudin) as anticoagulant. Lepirudin is the only known anticoagulant that does not interfere with complement activation. The results shown in Figure $7 \mathrm{C}$ demonstrate that SPIO NWs potently activate complement in fresh lepirudin plasma. CL-NWs-PEG showed only $26 \%$ of C3 opsonization of SPIO NWs in plasma. At the same time, CL-NWs-PEG-Ab (1 IgG/ particle), CL-NWs-PEG-Ab (46 IgG/particle), and CL-NWsPEG-Ab (160 IgG/particle) showed high C3 opsonization 

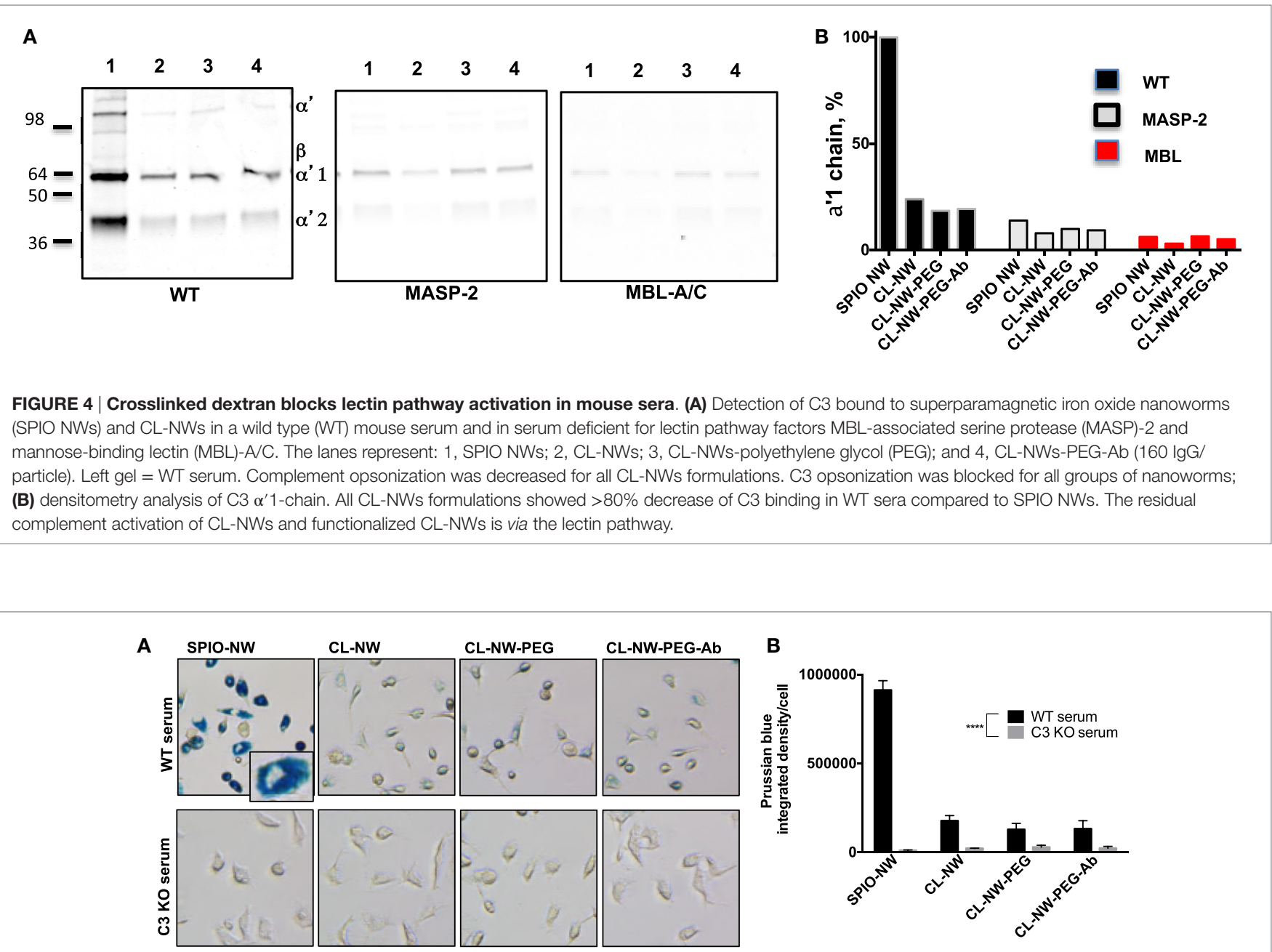

FIGURE 5 | Peritoneal macrophage uptake of superparamagnetic iron oxide nanoworms (SPIO NWs) and CL-NWs in mouse serum is complement dependent. (A) Prussian blue staining of iron uptake by cells. The peritoneal macrophages were incubated with SPIO NWs, CL-NWs, CL-NWs-polyethylene glycol (PEG), and CL-NWs-PEG-Ab (from left to right) in the presence of wild type (WT) serum (first row) or C3-deficient [knockout (KO)] serum (second row). All images were taken at 20x magnification and cropped to the same extent. Most of the Prussian blue stain is clearly intracellular as shown in the magnified insert;

(B) quantification of Prussian blue staining of iron in cells. The uptake of CL-NWs was $85 \%$ decreased compared with SPIO NWs $(n=20 ; p<0.0001)$. The uptake of nanoworms C3 KO serum was significantly less than in WT sera $(n=20 ; p<0.0001)$. For quantification and statistical analysis, see Section "Materials and Methods."

compared to SPIO NWs [46, 88, and $110 \%$ of C3 opsonization to SPIO NWs, respectively (Figure 7C)]. Therefore, the results in plasma are better correlated with C3 opsonization in vivo than in serum. At the same time, the relative levels of C3 opsonization were much higher in plasma than in vivo. Thus, plasma C3 opsonization of CL-NWs-PEG-Ab (46 IgG/particle) was $88 \%$ of SPIO NWs (Figure 7C), whereas in vivo C3 opsonization level of the same particle was $22 \%$ of SPIO NWs (Figure 7A). These discrepancies may be related to the dynamic differences in NW protein corona in vitro versus in vivo conditions in regulating complement activation (28). In summary, these data suggest that C3 assay in plasma is a better predictor of in vivo complement activation for $\mathrm{Ab}$-modified particles than in serum, but at the same time the opsonization efficiency in vitro does not fully correlate with the efficiency in vivo, likely due to differences in dynamics of protein interaction, corona formation, and complement activation.

Our studies demonstrate that surface modifications of nanoparticles have profound effect on complement C3 opsonization and the resultant immune uptake. Previous studies using PEGylated liposomes and nanoparticles showed loss of stealth properties after tethering of Abs and ligands (29-31) and that surface functionalization affects the cellular uptake (32-34). Nevertheless, the mechanistic studies explaining the effect of surface functionalizations were lacking and our study demonstrates for the first time the balance between surface functionalization, complement activation, and immune cell uptake. Immune system, including the complement system, is generally redundant, meaning that multiple pathways are utilized to recognize foreign epitopes. While the exact pathway by which surface $\mathrm{Ab}$ 
A

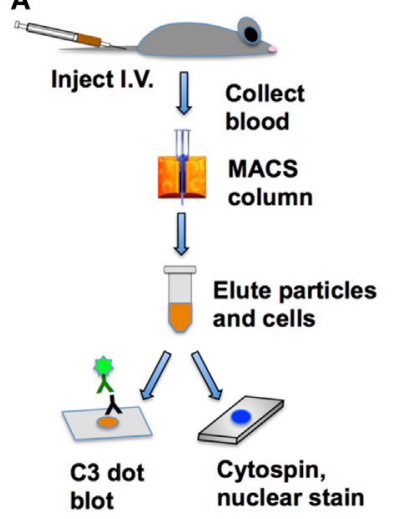

B

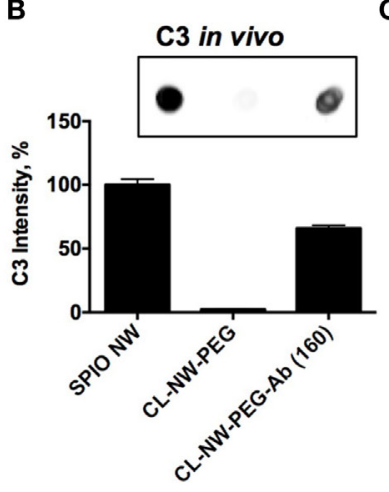

C
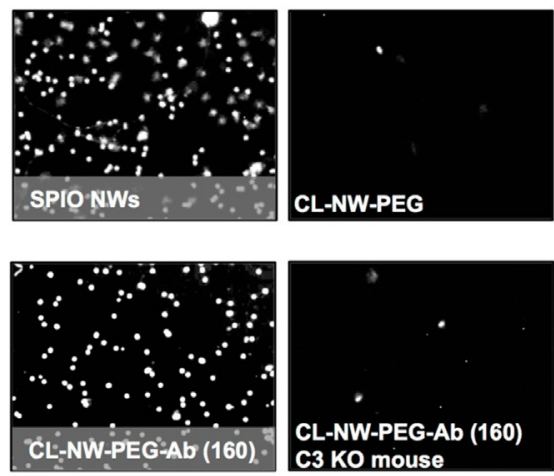

FIGURE 6 | Conjugated antibodies trigger complement activation and leukocyte uptake in vivo. (A) Nanoworms (NWs) were injected intravenously into mice and recovered from the blood 5 min post-injection. MIDI Max magnetic column trapped both free NWs and magnetically labeled leukocytes that took up NWs; (B) NWs were loaded on nitrocellulose membrane (same amount of Fe for all formulations) and analyzed for mouse C3. The image shows the C3 dot blot and the graph shows quantitative image analysis. There was no complement opsonization of CL-NWs-polyethylene glycol (PEG) but significant opsonization of CL-NWsPEG-Ab (160 lgG/particle); (C) microscopic images of slides after cytospin (nuclear Hoechst stain shows nucleated leukocytes) that were eluted from magnetic column. One representative cropped microscopic field is shown for each formulation. Each dot represents a leukocyte. A significant number of leukocytes took up particles after injection of superparamagnetic iron oxide nanoworms (SPIO NWs) and CL-NWs-PEGs-Ab. The uptake by leukocytes was abolished when CL-NWsPEG-Ab were injected into C3-deficient mouse (lower right).

A

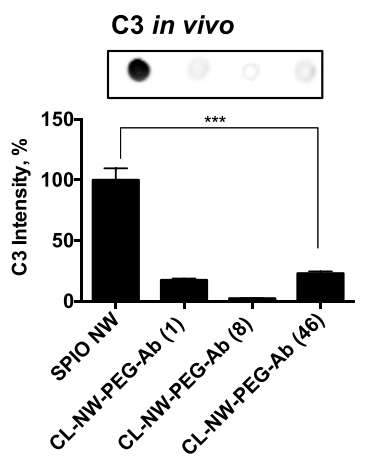

B

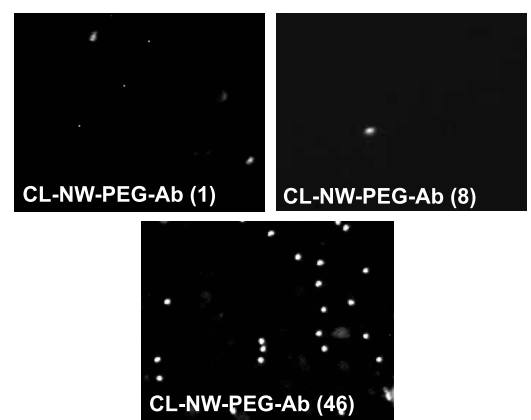

C C3 in plasma

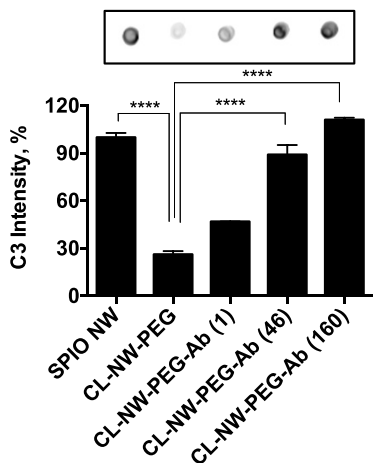

FIGURE 7 | Decreasing complement activation and leukocyte uptake in vivo by controlling number of antibodies per particle. (A) Complement opsonization in vivo of CL-NWs prepared with reduced number of lgG molecules per particle. Superparamagnetic iron oxide nanoworms (SPIO NWs) were used as a reference (100\%) of C3 levels. Complement opsonization was significantly decreased for CL-NWs-polyethylene glycol (PEG)-Ab with 1 lgG/particle, 8 lgG/particle, and $46 \mathrm{lgG} /$ particle compared to SPIO NWs ( $n=3 ; p<0.0001)$; (B) leukocyte uptake in vivo shows that CL-NWs-PEG-Ab with low number of IgG per particle show much lower leukocyte uptake than CL-NWs-PEG-Ab (160 lgG/particle, compared to Figure 6C); (C) complement C3 opsonization in fresh plasma in vitro. Particles were incubated in fresh plasma obtained with lepirudin as anticoagulant. C3 opsonization for CL-NWs-PEG-Ab was much higher in plasma than in serum (Figure 3) but also much higher than in vivo, suggesting that in vitro opsonization assay in plasma, while more representative than serum, still does not fully correlate with in vivo opsonization ( $p=0.0001$ for 46 lgG/particle and $160 \mathrm{Ab} /$ particle, respectively).

triggers complement activation needs to be elucidated further, the need to control complement activation via the number of conjugated Abs is an important aspect in the design of targetable nanomedicines.

Importantly, our study suggests that complement activation via surface immobilized $\mathrm{Ab}$ could be missed using in vitro assays in murine sera. Therefore, valid complement assays of nanoparticles should be used, at the very least using lepirudin plasma in vitro, but preferably in the animal in vivo. The lack of correlation between in vitro and in vivo assays adds to the lack of correlation in pathways of complement activation in mice and humans $(10,17)$. Mouse models are widely used in preclinical studies of biodistribution and toxicity of drug delivery systems. In addition, complement-mediated mechanisms of immune uptake are likely similar in mice and humans (10). Despite these, any extrapolation of mouse data on the immune recognition of nanocarriers to humans should be interpreted with caution. 


\section{MATERIALS AND METHODS}

\section{Materials}

All reagents used for NW synthesis including Fe salts and $20 \mathrm{kDa}$ (range 15-25 kDa) dextran were purchased from Sigma-Aldrich (St. Louis, MO, USA). Cell culture media were purchased from Corning Life Sciences (Corning, NY, USA). Copper grids (300 mesh) were purchased from Electron Microscopy Sciences (Hatfield, PA, USA). Anti-HER2 Ab Herceptin ${ }^{\circledR}$ was generously donated by the Pharmacy of the University of Colorado Cancer Center, Anschutz Medical Campus. Anti-mouse anti-C3 $\mathrm{Ab}$ was purchased from MP Biomedicals (Solon, OH, USA). IRDye 800CW-labeled secondary Abs were purchased from Li-COR Biosciences (Lincoln, NE, USA). Purified anti-mouse/ human $\mathrm{CD} 11 \mathrm{~b} \mathrm{Ab}$ was purchased from BioLegend (San Diego, CA, USA). Anti-mouse F4/80 Ab was purchased from Caltag Medsystems Ltd. (Buckingham, UK). Hoechst 33342 for nuclei staining was purchased from Thermo Fisher Scientific (Waltham, MA, USA). The Abs for each experiment were diluted according to the recommendations from manufacturers. Mouse sera deficient for $\mathrm{C} 3, \mathrm{MBL}-\mathrm{A} / \mathrm{C}, \mathrm{MBL}-\mathrm{A} / \mathrm{C} /$ factor $\mathrm{D}$, and factor $\mathrm{B}$ were collected from mice that were bred in an animal vivarium at the University of Colorado Anschutz Medical Campus according to the Institutional Animal Care and Use Committee (IACUC) approved breeding protocol. Recombinant human hirudin (lepirudin, catalog No. ACM154) was obtained from Aniara Diagnostica, LLC (West Chester, OH, USA), reconstituted in water to $1 \mu \mathrm{g} / \mu \mathrm{L}$ (160 antithrombin units $/ \mu \mathrm{L})$, and stored aliquoted at $-80^{\circ} \mathrm{C}$. Lepirudin anticoagulated plasma (hereafter plasma) was obtained by collecting blood through the cardiac puncture (final lepirudin concentration $3-4 \mu \mathrm{g} / \mathrm{mL}$ ) and centrifuging the tube at $2,000 \mathrm{~g}$ for $15 \mathrm{~min}$.

\section{Synthesis of SPIO and Crosslinked Nanoworms (CL-NWs)}

Nanoworms were synthesized by a modified one-pot Molday and MacKenzie (23) precipitation method as described earlier (16). Nanopure water $(30 \mathrm{~mL})$ was de-oxygenated with nitrogen gas and used to dissolve $6 \mathrm{~g}$ dextran (molecular weight $20 \mathrm{kDa}$, SigmaAldrich), $1.26 \mathrm{~g}$ Fe(III) chloride, and $0.498 \mathrm{~g}$ Fe(II) chloride in a round bottom flask. Next, $2.4 \mathrm{~mL}$ of cold $25 \%$ (v/v) ammonium hydroxide $\left(\mathrm{NH}_{4} \mathrm{OH}\right)$ was slowly added to the mixture of dextran and iron salts under nitrogen atmosphere with rapid stirring on ice. After formation of NWs, the mixture was heated at $80^{\circ} \mathrm{C}$ with stirring. After cooling, SPIO NWs were purified overnight using a $20 \mathrm{kDa}$ dialysis cassette (Thermo Fisher Scientific, Waltham, MA, USA) against double distilled water to remove free dextran. The particles were chemically crosslinked using 1-chloro-2, 3-epoxypropane (ECH) with sodium hydroxide as described before (16). SPIO NWs and CL-NWs were filtered through a $0.45 \mu \mathrm{Lm}$-pore filter (Millipore, Billerica, MA, USA) prior to use.

\section{Surface Modifications of CL-NWs}

CL-NWs were aminated by adding different concentrations of ammonium hydroxide at $4^{\circ} \mathrm{C}$ overnight to form CL-NWs- $\mathrm{NH}_{2}$ and dialyzed for $24 \mathrm{~h}$ to remove free $\mathrm{NH}_{4} \mathrm{OH}$. For the $\mathrm{Ab}$ conjugation, MAL-PEG-SVA (MAL-PEG-SVA, Laysan Bio) was reacted at excess with CL-NWs- $\mathrm{NH}_{2}$ at RT for $30 \mathrm{~min}$ to form CL-NWs-PEG-MAL or CL-NWs-PEG. Anti-HER2 Ab Herceptin $^{\circledR}$ was reacted in the last step with MAL-PEG to form CL-NWs-PEG-Ab. NWs were purified by ultracentrifugation at $55,000 \mathrm{rpm}$, filtered through a $0.45 \mu \mathrm{m}$ pore filter, and finally, stored in Dulbecco's phosphate-buffered saline (DPBS) at $\mathrm{pH} 7.4$ before use.

\section{Characterization of NWs}

Transmission electron microscopy imaging was conducted to visualize the NWs using FEI Tecnai Spirit BioTwin electron microscope (Electron Microscopy Facility at the University of Colorado Boulder) at a $100 \mathrm{kV}$ working voltage. Size measurements were done in DPBS and zeta potential measurements were done in $0.1 \times$ DPBS at RT using a Zetasizer Nano ZS (Malvern Instruments Ltd., Malvern, UK). The intensity-weighted size distribution peak value was used to report hydrodynamic diameters of the NWs. To quantify the $\mathrm{Ab}$ on the NWs, known amount of CL-NWsPEG-Ab $(0.2 \mu \mathrm{g} F)$ was applied in triplicates onto a $0.2 \mu \mathrm{m}$ pore nitrocellulose membrane (Bio-Rad). Standard dilutions of free trastuzumab were also applied onto the membrane to generate a standard curve. The membrane was blocked with $5 \%(\mathrm{w} / \mathrm{w})$ non-fat dry milk in PBS-T (DPBS with $0.1 \%$ Tween $^{\circledR} 20$ ) for $1 \mathrm{~h}$ at RT and probed with IRDye 800CW-labeled antihuman Ab. The membrane was scanned with Odyssey infrared imager (Li-COR Biosciences, Lincoln, NE, USA). The integrated dot intensity in the scanned images was determined from 16-bit grayscale images using ImageJ software and plotted using Prism 6 software (GraphPad Software, Inc., La Jolla, CA, USA) to determine the number of $\mathrm{Ab}$ molecules per spot using the calibration curve. Concentration of particles per milligram $\mathrm{Fe}$ was determined with NanoSight (Malvern Instruments) and Fe concentration was determined with ferrozine iron assay as described before (16). The number of Abs per NW was determined after dividing the number of Abs per spot by the number of NWs per spot.

\section{Protein Binding Assay}

Superparamagnetic iron oxide and CL-NWs $(10 \mu \mathrm{L}$ of $1 \mathrm{mg} / \mathrm{mL})$ were incubated with $30 \mu \mathrm{L}$ of mouse serum or lepirudin plasma for $15 \mathrm{~min}$ at RT. At the end of incubation, particles were washed three times with $1 \times$ PBS by centrifugation at $100,000 \mathrm{~g}$ at $4^{\circ} \mathrm{C}$ using Beckman Optima TLX ultracentrifuge. The pellets were resuspended in $20 \mu \mathrm{L}$ DPBS, and the concentration of Fe in each sample was normalized with ferrozine iron assay as described before (16). For complement C3 western blot, $10 \mu \mathrm{L}$ aliquots were used for gel electrophoresis. The samples were mixed with loading buffer [denaturing buffer containing $100 \mathrm{mM}$ Tris, 20\% glycerol, $4 \%$ SDS, 5\% (v/v) 2-mercaptoethanol, $0.02 \%$ bromophenol blue] and then boiled at $95^{\circ} \mathrm{C}$ for $5 \mathrm{~min}$. After cooling for $5 \mathrm{~min}$, the samples and marker proteins (Precision Plus Proteins Dual Color Standards from Bio-Rad) were loaded onto Mini-PROTEAN TGX Gels (Bio-Rad, Hercules, CA, USA) and separated at $50 \mathrm{~V}$ for $5 \mathrm{~min}$ and then $100 \mathrm{~V}$ for $90 \mathrm{~min}$. Gels were then transferred to nitrocellulose membranes using the Mini Trans-Blot cell system overnight at $50 \mathrm{~V}$ at $4^{\circ} \mathrm{C}$. For $\mathrm{C} 3$ dot blot, $2 \mu \mathrm{L}$ aliquots were applied in triplicates onto a nitrocellulose membrane. 
The membranes were blocked using 5\% non-fat dry milk in DPBS-T (DPBS with $0.1 \%$ Tween $^{\circledR} 20$ ) at RT for $1 \mathrm{~h}$, probed with corresponding primary Abs at RT for $1 \mathrm{~h}$, followed by washing the membranes $3 \times$ with DPBS-T, and finally, $1 \mathrm{~h}$ incubation with the corresponding IRDye 800CW-labeled secondary Abs against the primary $\mathrm{Ab}$ species (see Materials). The membranes after immunoblotting were visualized using an Odyssey infrared imager. The integrated dot intensity in the scanned images was quantitatively analyzed using ImageJ software and plotted with Prism 6 software as described above.

\section{Uptake of NWs In Vitro, Prussian Blue Staining, and Quantification}

Human breast cancer cell line SKBR-3 cells were maintained in McCoy's 5A medium (ATCC) supplemented with 10\% fetal bovine serum. Mouse peritoneal macrophages were obtained by peritoneal lavage with $5 \mathrm{~mL}$ ice cold PBS, post-mortem. For experiment, cells were plated into 96-well plate. For uptake experiments, NWs were preincubated for $15 \mathrm{~m}$ with WT mouse sera or $\mathrm{C} 3 \mathrm{KO}$ sera and added at $0.1 \mathrm{mg} / \mathrm{mL}$ Fe concentration to cells for $6 \mathrm{~h}$. After the incubation, cells were washed using DPBS for three times, fixed in $4 \%$ paraformaldehyde at $4^{\circ} \mathrm{C}$ overnight, and then stained using Prussian blue for $1 \mathrm{~h}$. Prussian blue staining is the standard method for detecting iron in cells and tissues, and has been extensively used for detecting uptake of iron oxide nanoparticles by our group and others $(35,36)$. The method is based on formation of insoluble, blue colored coordination complexes between $\mathrm{Fe}^{3+}$ and potassium ferrocyanide. Maghemite crystals of SPIO contain both $\mathrm{Fe}^{3+}$ and $\mathrm{Fe}^{2+}$, and the surface of crystals is always oxidized to $\mathrm{Fe}^{3+}$, so the complete degradation of nanoparticles is not required for the staining to work. In order to quantify the blue color of the complexes inside the cells, TIFF RGB images of stained cells were acquired with a Nikon Eclipse E600 microscope using same exposure and magnification. The images were combined into a gallery with Adobe Photoshop and color balance was adjusted with a Level tool to make the cell-free area white. The gallery image was exported into ImageJ, converted into YUV color space, and thresholded for blue and green components using a Threshold Color plugin. Then, the image was converted into a 16-bit gray scale and inverted. The background was completely subtracted with a Math Subtraction tool. A ROI was drawn around each cell and integrated pixel density was measured. The data were plotted as means and SD using Prism software. An average of at least 20 cells was used.

\section{Nanoparticle Uptake In Vivo}

Wild type and $\mathrm{C3}^{-/-}$mice (Jax Laboratories: B6.129S4-C3 ${ }^{\text {tmlCrr/ }} \mathrm{J}$ ) were bred in house according to the approval by University of Colorado Animal Protocol Committee. NWs were injected as a $5 \mathrm{mg} / \mathrm{kg}$ bolus via tail vein into WT and C3 KO mice (8 weeks

\section{REFERENCES}

1. Moghimi SM, Hunter AC, Murray JC. Long-circulating and targetspecific nanoparticles: theory to practice. Pharmacol Rev (2001) 53(2): $283-318$. of age, females). Following the injection (5 min), mice were sacrificed and the blood was drawn via cardiac puncture using heparin as anticoagulant. Blood was applied on Miltenyi Mini MACS magnetic column (Miltenyi Biotech) and the trapped cells and particles were washed extensively with PBS. The particles and magnetic leukocytes were then eluted from the column. The cells were pelleted with tabletop Eppendorf centrifuge and the particles in the supernatant were further concentrated with ultracentrifuge for C3 dot-blot assay. The cell pellet was resuspended in $200 \mu \mathrm{L}$ PBS and the cells were concentrated on slides using Shandon Cytospin 4 centrifuge (Thermo Fischer), fixed with 10\% (v/v) formalin in PBS, and stained with Hoechst dye (Thermo Fischer) to enable leukocyte nuclei visualization. The images were taken with Nikon Eclipse E600 fluorescent microscope using DAPI filter at low $(40 \times)$ magnification.

\section{Statistical Analysis}

The statistical analysis was performed using Prism 6 software. The differences between means of experimental groups were analyzed using a two-tailed parametric $t$-test assuming 95\% confidence interval. Data shown as means $\pm \mathrm{SD}$. Differences in all data are shown as ${ }^{\star} p<0.05 ;{ }^{* *} p<0.001 ;{ }^{* * *} p<0.0001$.

\section{ETHICS STATEMENT}

This study was carried out in accordance with the guidelines of the University of Colorado Office of Animal Care. The protocol was approved by the University of Colorado IACUC.

\section{AUTHOR CONTRIBUTIONS}

GW designed and performed experiments, and analyzed data. JG performed experiments. SI performed experiments. BB performed experiments. NB analyzed data and provided reagents. VH analyzed data and provided reagents. SM designed experiments, analyzed data, and edited the paper. DS designed experiments, analyzed data, and wrote the paper.

\section{FUNDING}

The study was funded by the University of Colorado Denver startup and NIH grants EB022040 and CA194058-01A1 to DS, and the Danish Agency for Science, Technology and Innovation (Det Strategiske Forskningsråd), reference 09-065746 as well as RiboBio Co. Ltd. (Guangzhou, China) to SM.

\section{SUPPLEMENTARY MATERIAL}

The Supplementary Material for this article can be found online at http://journal.frontiersin.org/article/10.3389/fimmu. 2017.00151/full\#supplementary-material.

2. Ricklin D, Hajishengallis G, Yang K, Lambris JD. Complement: a key system for immune surveillance and homeostasis. Nat Immunol (2010) 11(9):785-97. doi:10.1038/ni.1923

3. Janssen BJC, Huizinga EG, Raaijmakers HCA, Roos A, Daha MR, NilssonEkdahl K, et al. Structures of complement component C3 provide insights into 
the function and evolution of immunity. Nature (2005) 437(7058):505-11. doi:10.1038/nature04005

4. Arima Y, Kawagoe M, Toda M, Iwata H. Complement activation by polymers carrying hydroxyl groups. ACS Appl Mater Interfaces (2009) 1(10):2400-7. doi:10.1021/am9005463

5. Lemarchand C, Gref R, Passirani C, Garcion E, Petri B, Muller R, et al. Influence of polysaccharide coating on the interactions of nanoparticles with biological systems. Biomaterials (2006) 27(1):108-18. doi:10.1016/ j.biomaterials.2005.04.041

6. Helmy KY, Katschke KJ Jr, Gorgani NN, Kljavin NM, Elliott JM, Diehl L, et al. CRIg: a macrophage complement receptor required for phagocytosis of circulating pathogens. Cell (2006) 124(5):915-27. doi:10.1016/j.cell.2005. 12.039

7. Taylor PR, Martinez-Pomares L, Stacey M, Lin HH, Brown GD, Gordon S. Macrophage receptors and immune recognition. Annu Rev Immunol (2005) 23:901-44. doi:10.1146/annurev.immunol.23.021704.115816

8. Peng Q, Li K, Sacks SH, Zhou W. The role of anaphylatoxins C3a and C5a in regulating innate and adaptive immune responses. Inflamm Allergy Drug Targets (2009) 8(3):236-46. doi:10.2174/187152809788681038

9. Dobrovolskaia MA, McNeil SE. Immunological properties of engineered nanomaterials. Nat Nanotechnol (2007) 2(8):469-78. doi:10.1038/nnano. 2007.223

10. Inturi S, Wang G, Chen F, Banda NK, Holers VM, Wu L, et al. Modulatory role of surface coating of superparamagnetic iron oxide nanoworms in complement opsonization and leukocyte uptake. ACS Nano (2015) 9(11):10758-68. doi:10.1021/acsnano.5b05061

11. Moghimi SM. Cancer nanomedicine and the complement system activation paradigm: anaphylaxis and tumour growth. JControl Release (2014) 190:556-62. doi:10.1016/j.jconrel.2014.03.051

12. Hamad I, Al-Hanbali O, Hunter AC, Rutt KJ, Andresen TL, Moghimi SM. Distinct polymer architecture mediates switching of complement activation pathways at the nanosphere-serum interface: implications for stealth nanoparticle engineering. ACS Nano (2010) 4(11):6629-38. doi:10.1021/ nn101990a

13. Pham CT, Mitchell LM, Huang JL, Lubniewski CM, Schall OF, Killgore JK, et al. Variable antibody-dependent activation of complement by functionalized phospholipid nanoparticle surfaces. J Biol Chem (2011) 286(1):123-30. doi:10.1074/jbc.M110.180760

14. Figuerola A, Di Corato R, Manna L, Pellegrino T. From iron oxide nanoparticles towards advanced iron-based inorganic materials designed for biomedical applications. Pharmacol Res (2010) 62(2):126-43. doi:10.1016/j. phrs.2009.12.012

15. Gupta AK, Gupta M. Synthesis and surface engineering of iron oxide nanoparticles for biomedical applications. Biomaterials (2005) 26(18):3995-4021. doi:10.1016/j.biomaterials.2004.10.012

16. Wang G, Inturi S, Serkova NJ, Merkulov S, McCrae K, Russek SE, et al. High-relaxivity superparamagnetic iron oxide nanoworms with decreased immune recognition and long-circulating properties. ACS Nano (2014) 8(12):12437-49. doi:10.1021/nn505126b

17. Banda NK, Mehta G, Chao Y, Wang G, Inturi S, Fossati-Jimack L, et al. Mechanisms of complement activation by dextran-coated superparamagnetic iron oxide (SPIO) nanoworms in mouse versus human serum. Part Fibre Toxicol (2014) 11(1):64. doi:10.1186/s12989-014-0064-2

18. Wang G, Chen F, Banda NK, Holers VM, Wu L, Moghimi SM, et al. Activation of human complement system by dextran-coated iron oxide nanoparticles is not affected by dextran/Fe ratio, hydroxyl modifications, and crosslinking. Front Immunol (2016) 7:418. doi:10.3389/fimmu.2016.00418

19. Iwaki D, Kanno K, Takahashi M, Endo Y, Lynch NJ, Schwaeble WJ, et al. Small mannose-binding lectin-associated protein plays a regulatory role in the lectin complement pathway. J Immunol (2006) 177(12):8626-32. doi:10.4049/ jimmunol.177.12.8626

20. Takahashi M, Iwaki D, Kanno K, Ishida Y, Xiong J, Matsushita M, et al. Mannose-binding lectin (MBL)-associated serine protease (MASP)-1 contributes to activation of the lectin complement pathway. J Immunol (2008) 180(9):6132-8. doi:10.4049/jimmunol.180.9.6132

21. Iwaki D, Kanno K, Takahashi M, Endo Y, Matsushita M, Fujita T. The role of mannose-binding lectin-associated serine protease-3 in activation of the alternative complement pathway. J Immunol (2011) 187(7):3751-8. doi:10.4049/ jimmunol.1100280
22. Takahashi M, Ishida Y, Iwaki D, Kanno K, Suzuki T, Endo Y, et al. Essential role of mannose-binding lectin-associated serine protease-1 in activation of the complement factor D. J Exp Med (2010) 207(1):29-37. doi:10.1084/ jem. 20090633

23. Molday RS, MacKenzie D. Immunospecific ferromagnetic iron-dextran reagents for the labeling and magnetic separation of cells. J Immunol Methods (1982) 52(3):353-67. doi:10.1016/0022-1759(82)90007-2

24. Dunkelberger JR, Song WC. Complement and its role in innate and adaptive immune responses. Cell Res (2010) 20(1):34-50. doi:10.1038/cr.2009.139

25. Lachmann PJ. Preparing serum for functional complement assays. J Immunol Methods (2010) 352(1-2):195-7. doi:10.1016/j.jim.2009.11.003

26. van Dijk H, Rademaker PM, Willers JM. Estimation of classical pathway of mouse complement activity by use of sensitized rabbit erythrocytes. J Immunol Methods (1980) 39(3):257-68. doi:10.1016/0022-1759(80)90060-5

27. Ratelade J, Verkman AS. Inhibitor(s) of the classical complement pathway in mouse serum limit the utility of mice as experimental models of neuromyelitis optica. Mol Immunol (2014) 62(1):104-13. doi:10.1016/j.molimm.2014.06.003

28. Chen F, Wang G, Griffin JI, Brenneman B, Banda NK, Holers VM, et al. Complement proteins bind to nanoparticle protein corona and undergo dynamic exchange in vivo. Nat Nanotechnol (2016). doi:10.1038/ nnano.2016.269

29. Cheng WW, Allen TM. Targeted delivery of anti-CD19 liposomal doxorubicin in B-cell lymphoma: a comparison of whole monoclonal antibody, Fab' fragments and single chain Fv. J Control Release (2008) 126(1):50-8. doi:10.1016/ j.jconrel.2007.11.005

30. Iden DL, Allen TM. In vitro and in vivo comparison of immunoliposomes made by conventional coupling techniques with those made by a new postinsertion approach. Biochim Biophys Acta (2001) 1513(2):207-16. doi:10.1016/ S0005-2736(01)00357-1

31. McNeeley KM, Annapragada A, Bellamkonda RV. Decreased circulation time offsets increased efficacy of PEGylated nanocarriers targeting folate receptors of glioma. Nanotechnology (2007) 18(38):Artn 385101. doi:10.1088/0957-4484/18/38/385101

32. Jiang XE, Dausend J, Hafner M, Musyanovych A, Rocker C, Landfester K, et al. Specific effects of surface amines on polystyrene nanoparticles in their interactions with mesenchymal stem cells. Biomacromolecules (2010) 11(3):748-53. doi:10.1021/bm901348z

33. Ekkapongpisit M, Giovia A, Follo C, Caputo G, Isidoro C. Biocompatibility, endocytosis, and intracellular trafficking of mesoporous silica and polystyrene nanoparticles in ovarian cancer cells: effects of size and surface charge groups. Int J Nanomedicine (2012) 7:4147-58. doi:10.2147/IJN.S33803

34. Villanueva A, Canete M, Roca AG, Calero M, Veintemillas-Verdaguer S, Serna CJ, et al. The influence of surface functionalization on the enhanced internalization of magnetic nanoparticles in cancer cells. Nanotechnology (2009) 20(11):115103. doi:10.1088/0957-4484/20/11/115103

35. Raynal I, Prigent P, Peyramaure S, Najid A, Rebuzzi C, Corot C. Macrophage endocytosis of superparamagnetic iron oxide nanoparticles: mechanisms and comparison of ferumoxides and ferumoxtran-10. Invest Radiol (2004) 39(1):56-63. doi:10.1097/01.rli.0000101027.57021.28

36. Chao Y, Karmali PP, Mukthavaram R, Kesari S, Kouznetsova VL, Tsigelny IF, et al. Direct recognition of superparamagnetic nanocrystals by macrophage scavenger receptor SR-AI. ACS Nano (2013) 7(5):4289-98. doi:10.1021/ nn400769e

Conflict of Interest Statement: The authors declare that the research was conducted in the absence of any commercial or financial relationships that could be construed as a potential conflict of interest.

The reviewer YL declared a shared affiliation, though no other collaboration, with several of the authors to the handling Editor, who ensured that the process nevertheless met the standards of a fair and objective review.

Copyright $\odot 2017$ Wang, Griffin, Inturi, Brenneman, Banda, Holers, Moghimi and Simberg. This is an open-access article distributed under the terms of the Creative Commons Attribution License (CC BY). The use, distribution or reproduction in other forums is permitted, provided the original author(s) or licensor are credited and that the original publication in this journal is cited, in accordance with accepted academic practice. No use, distribution or reproduction is permitted which does not comply with these terms. 